\title{
Simple Screening Method for Staurosporine in Bacterial Cultures using Liquid Chromatography-Tandem Mass Spectrometry
}

\author{
Amitha K. Hewavitharana ${ }^{4}$, P. Nicholas Shaw', Yi K. Ng², John A. Fuerst ${ }^{2}$ \\ 'School of Pharmacy, The University of Queensland, Brisbane, Queensland 4072, Australia \\ ${ }^{2}$ School of Molecular and Microbial Sciences, The University of Queensland, Brisbane, Queensland 4072, Australia
}

\begin{abstract}
Staurosporine has been shown to possess an array of important biological properties such as anti-fungal, anti-bacterial, antihypertensive and anti-cancer. Methods available to screen any plant or bacterial extract for this compound are lengthy and laborious. We present here a simple HPLC-MS-MS method for the highly selective identification of Staurosporine in various strains of the marine sponge-derived bacterium Salinispora. Gradient elution using acetonitrile/water/ammonium acetate was used to separate Staurosporine from the matrix and positive ionelectrospray mass spectrometry was used for detection and confirmation. Presence of Staurosporine in bacterial extracts was confirmed by matching retention times and parent-daughter ion mass spectra (using Multiple Reaction Monitoring, MRM mode) of the standard Staurosporine with those of the bacterial extracts. Detection of Staurosporine was achieved down to $11 \mathrm{ng} / \mathrm{mL}$ bacterial extract. This method can be easily adapted to screen any plant extract for Staurosporine. The simplicity and the speed of this method make it possible for the analyst to screen a large number of extracts before embarking on lengthy and costly isolation and purification of Staurosporine in a selected few.
\end{abstract}

Keywords: HPLC-MS-MS; Tandem mass spectrometry; Salinispora; Staurosporine; Screening; Detection

\section{Introduction}

Staurosporine (antibiotic AM-2282) was discovered in 1977 from bacterium Streptomyces staurosporeus (Ōmura et al., 1977). The chemical structure of Staurosporine was determined in 1994 by X-ray crystallography (Funato et al., 1994) and the synthesis was completed soon after (Link et al., 1995). In addition to being an antibiotic, Staurosporine has been shown to possess an array of important biological properties such as anti-fungal (Ōmura et al., 1977), anti-hypertensive (Hachisu et al., 1989) and platelet aggregation inhibition (Schachtele et al., 1988). However, its anti-cancer properties, based on the inhibition of protein kinase $\mathrm{C}$ were the most important aspect of its biological profile (Yamamoto et al., 1989; Bradshaw et al., 1992; Lin et al., 1992). Numerous studies continue to be published on anticancer properties of Staurosporine: over 2000 hits on Staurosporine within the last five years in Web of Science.

Although there have been continued interest in isolating Staurosporine from a variety of bacterial strains (Park et al., 2006; Wu et al., 2006), to date, there is no method available for screening the bacterial extracts for the presence of Staurosporine. The method used in published studies so far (Park et al., 2006; Wu et al., 2006) involved lengthy multistep extractions and/or multiple chromatographic separations to isolate a substantial amount of the pure Staurosporine followed by nuclear magnetic resonance (nmr) spectroscopy to confirm the structure. This type of procedure prevents the researcher from screening many extracts. A quick screening method will enable the researchers to screen a variety of extracts and exclude the ones that do not contain Staurosporine before embarking on a lengthy and costly cleanup and isolation. It will also enable the researcher to obtain an approximate concentration of Staurosporine therefore exclude those extracts that are scarce in Staurosporine.

We describe here a simple screening method that can also serve as a semi-quantitative method for Staurosporine. The method uses four different parameters (retention time, molecular ion mass, ion masses of two fragments) to confirm the presence of Staurosporine in extracts therefore the researcher can invest on a large scale extraction and isolation with confidence. Recent developments in liquid chromatography- tandem mass spectroscopy (LC-MS-MS) have provided an extremely selective tool for confirmation of the presence of a molecule in a complex matrix without isolating it (Hewavitharana et al., 2007). The Multiple Reaction Monitoring (MRM) mode of tandem mass spectrometry used in this work holds great potential for confirmation studies due to its superior sensitivity and the specificity. Although LC-MS has been used to screen Staurosporine in a recent study (Jensen et al., 2007) the single mass spectrometric method lacks the sensitivity and the added specificity that is brought about by the tandem MS and the MRM mode used here. While an LC-MS method can identify a wrong compound that elutes at the same retention time and have the same molecular mass (or one of its fragments have the same mass) as the compound of interest, the same happening with LC-MS-MS is almost impossible because to produce an MRM signal the said compound must also produce the same fragments as the compound of interest. Monitoring more than one fragment through MRM further eliminates the possibility of mis-identification. Further, the sensitivity of LC-MRM method is a few hundred times more sensitive than that of the LC-MS method, thus eliminate the need for lengthy sample extraction and concentration

*Corresponding author: Amitha K. Hewavitharana, School of Pharmacy, The University of Queensland, Brisbane, Queensland 4072, Australia, Tel: +61-7-3365-8853; Fax: +61-7-3365-1688; E-mail: a.hewavitharana@pharmacy.uq.edu.au

Received November 10, 2009; Accepted December 20, 2009; Published December 21, 2009

Citation: Hewavitharana AK, Shaw PN, Ng YK, Fuerst JN (2009) Simple Screening Method for Staurosporine in Bacterial Cultures using Liquid Chromatography-Tandem Mass Spectrometry. J Bioanal Biomed 1: 001004. doi:10.4172/1948-593X.1000001

Copyright: ( $) 2009$ Hewavitharana AK, et al. This is an open-access article distributed under the terms of the Creative Commons Attribution License, which permits unrestricted use, distribution, and reproduction in any medium, provided the original author and source are credited. 
Citation: Hewavitharana AK, Shaw PN, Ng YK, Fuerst JN (2009) Simple Screening Method for Staurosporine in Bacterial Cultures using Liquid Chromatography-Tandem Mass Spectrometry. J Bioanal Biomed 1: 001-004. doi:10.4172/1948-593X.1000001

procedures used with LC-MS method (Jensen et al., 2007).

\section{Materials and Methods}

\section{Instrumentation and materials}

Separations were carried out using an Agilent binary HPLC system consisting of an Agilent 1100 LC pump, an Agilent 1100 well plate autosampler, and an Agilent ZORBAX SB-C18 (2.1 x $150 \mathrm{~mm}, 3.5 \mu \mathrm{m})$ HPLC column (Agilent Technologies, Santa Clara, CA, USA). An API 3000 tandem mass spectrometer equipped with a turbo ion spray interface and supported by Analyst 1.4 software (Applied Biosystems, Foster City, CA, USA) was used to detect the compounds and process data.

Staurosporine was purchased from Sigma (St. Louis, MO, USA). All solvents used were of HPLC grade.

\section{Sample preparation}

Salinispora spp. (M101, M102, M403, M412, M413, SW02, SW15, YKPC1, YKPC2, YKPC3, YKPC4, YKAPL1, YKAPL2, YKAPL3 and YKAPL4) were maintained in SYP broth cultures at $28^{\circ} \mathrm{C}$ for 4 weeks. A $6 \mathrm{ml}$ volume of broth culture, from each Salinispora strain, was transferred to a falcon tube. A $6 \mathrm{ml}$ volume of ethylacetate was added to each tube. The brothethylacetate mixture was incubated at room temperature for 90 min with gentle rotation. Ethyl acetate forms a clear layer, the upper layer, in the falcon tube. A $3 \mathrm{ml}$ volume of ethyl acetate was transferred to a fresh tube and dried using a vacuum pump centrifuge. The extracts were re-suspended in $300 \mu \mathrm{L}$ of $20 \% \mathrm{v} /$ v methanol. The samples were stored at $-20^{\circ} \mathrm{C}$, and filtered using a $0.22 \mu \mathrm{m}$ pore size immediately before HPLC analysis. A blank extract was also prepared in a similar manner using sterile culture medium.

\section{HPLC separation}

A $20 \mu \mathrm{L}$ aliquot of each sample was injected onto the HPLC column using the autosampler. Various volumes of a standard solution of $1 \mu \mathrm{M}$ Staurosporine, and the blank extract were also injected in a similar manner. A binary solvent gradient consisting of a solution of ammonium acetate in water $(5 \mathrm{mM}$; A) and a solution of ammonium acetate in acetonitrile $(5 \mathrm{mM}$; B) was used for all separations The gradient was started after the column had been equilibrated using a mobile phase of $60 \% \mathrm{v} / \mathrm{v} \mathrm{A}$ and $40 \% \mathrm{v} / \mathrm{v}$ B for $15 \mathrm{~min}$. The composition of the mobile phase remained the same for the first 5 min after injection and then changed from $40 \% \mathrm{v} / \mathrm{v}$ B to $100 \% \mathrm{v} / \mathrm{v}$ B over the next $10 \mathrm{~min}$ and was then returned to the starting composition of $40 \% \mathrm{~B}$ over the next 5 min. The column was then re-equilibrated using $40 \%$ v/v B for $15 \mathrm{~min}$ before the next sample was injected. The total run time was $35 \mathrm{~min}$. The mobile phase flow rate was $200 \mu \mathrm{L} /$ $\min$.

\section{MRM positive ion mode mass spectrometry}

The MS signal in MRM mode is produced by the specific transition of molecular ion $\rightarrow$ fragment. The fragmentation of the protonated molecular ion $467 \mathrm{~m} / \mathrm{z}$ ion $\rightarrow 338 \mathrm{~m} / \mathrm{z}$ ion and 467 $\mathrm{m} / \mathrm{z}$ ion $\rightarrow 130 \mathrm{~m} / \mathrm{z}$ ion were monitored during the chromatographic run. Following are the parameters optimized to obtain the highest possible sensitivity for Staurosporine:

An ion spray voltage (IS) of $5000 \mathrm{~V}$, orifice/ declustering po- tential (DP) of $51 \mathrm{~V}$, ring/ focusing potential (FP) of $250 \mathrm{~V}$, entrance potential $(\mathrm{EP})$ of $10 \mathrm{~V}$, collision energy $(\mathrm{CE})$ of $23 \mathrm{~V}$ and collision exit potential (CXP) of $10 \mathrm{~V}$ were used for all experiments. Collision gas (CAD) flow and the nebulizer gas (NEB) flow were maintained at 8 and curtain gas flow (CUR) was $12 \mathrm{~L} /$ $\mathrm{min}$. Temperature of the ion spray was maintained at $300^{\circ} \mathrm{C}$. Dwell time of $150 \mathrm{msec}$ was used in both transitions. Resolution of both Q1 and Q3 was $1 \mathrm{amu}$.

\section{MS2 positive ion mode mass spectrometry}

To obtain the fragmentation pattern of the protonated molecular ion the same conditions as in MRM mode were employed except that the fragmentation of $467 \mathrm{~m} / \mathrm{z}$ ion was monitored within the range 50-520 amu, during the chromatographic run.

\section{Results and Discussion}

Staurosporine is an indolo [2,3-a]carbazole alkaloid, the structure of which is shown in Figure 1. The positive ion ESI-MS of Staurosporine produced the protonated molecular ion at $467 \mathrm{amu}$ and the ESI-MS-MS produced two major fragments at $338 \mathrm{amu}$ and 130 amu. Fragmentation study of Staurosporine has been carried out previously by electron impact (EI) mass spectrometry (Yang and Cordell, 1997) in which the mechanism for the production of 338 amu ion was elucidated. The ESI mass spectrometry in our study has produced two major fragments: 338 amu and $130 \mathrm{amu}$. Figure 2 shows the chromatogram (monitored using MS2 mode) and the fragmentation spectrum of standard Staurosporine showing the two major fragments.

The chromatograms monitored using MRM mode showed that chromatograms of only 4 out of 15 of the extracts contained a peak that eluted with the same retention time as the chromatograms of the standard Staurosporine (data not shown). These

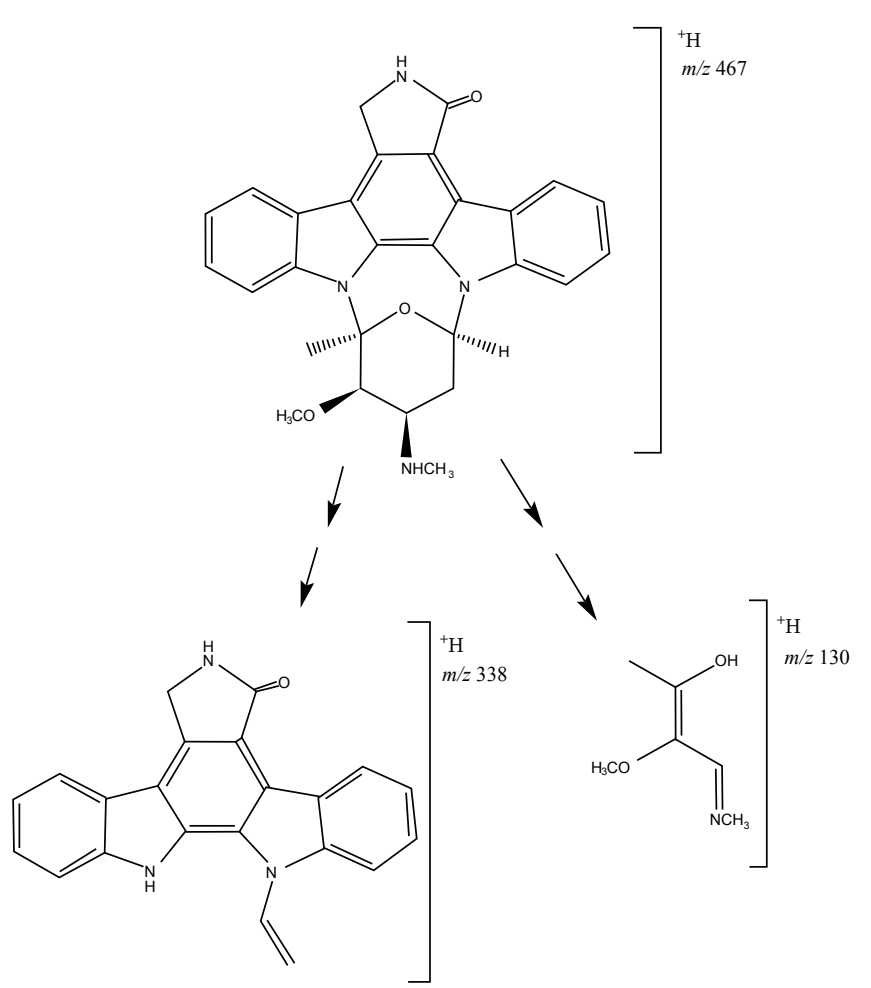

Figure 1: Sructure of Staurosporine showing the fragments produced with positive ion ESI-MS-MS.

Volume 1(1) : 001-004 (2009) - 002 


\section{Journal of Bioanalysis \& Biomedicine - Open Access}

\section{JBABM/Vol.1 Issue 1}
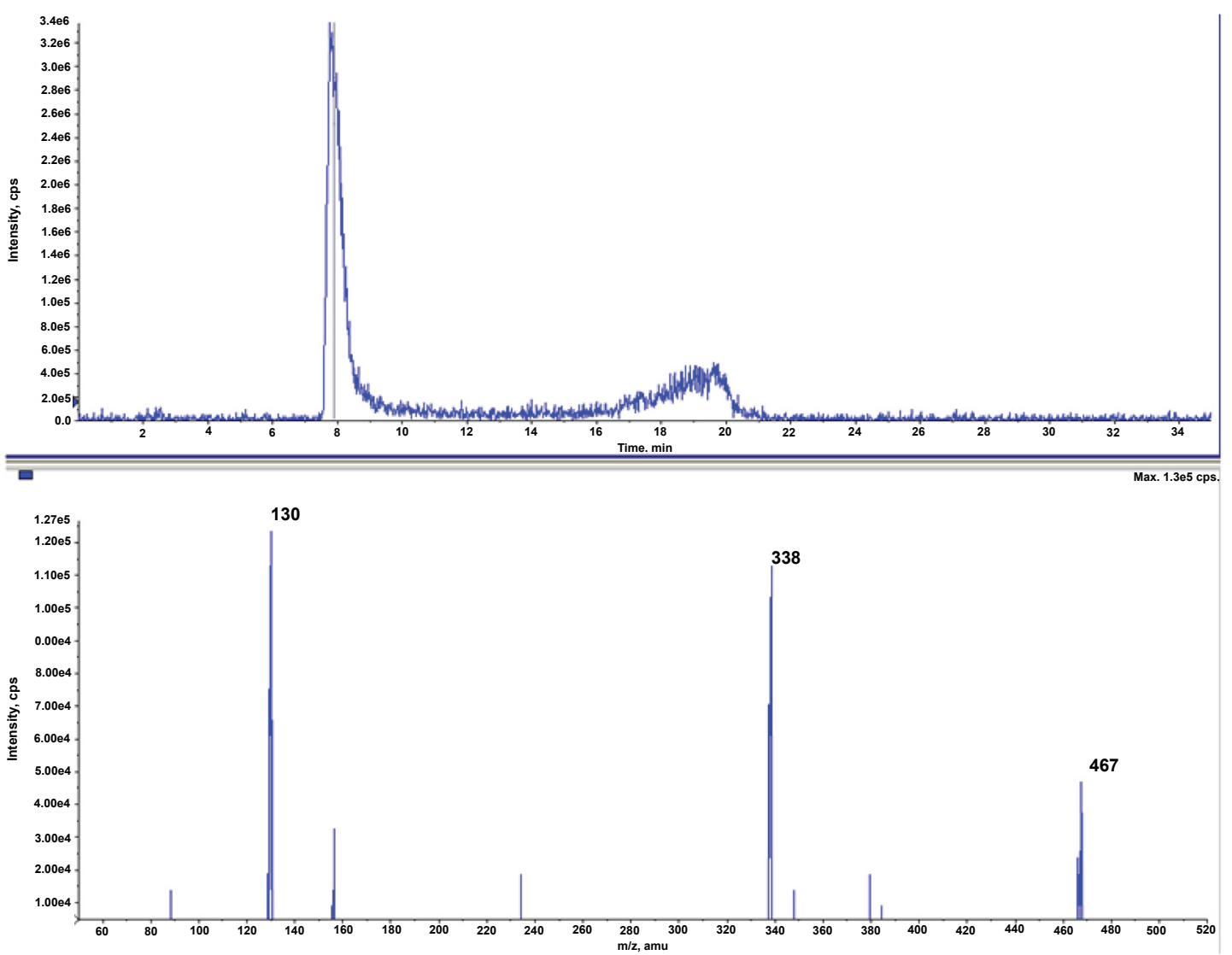

Figure 2: Chromatogram of Staurosporine standard monitored using MS2 mode and the fragmentation pattern for the Staurosporine peak.
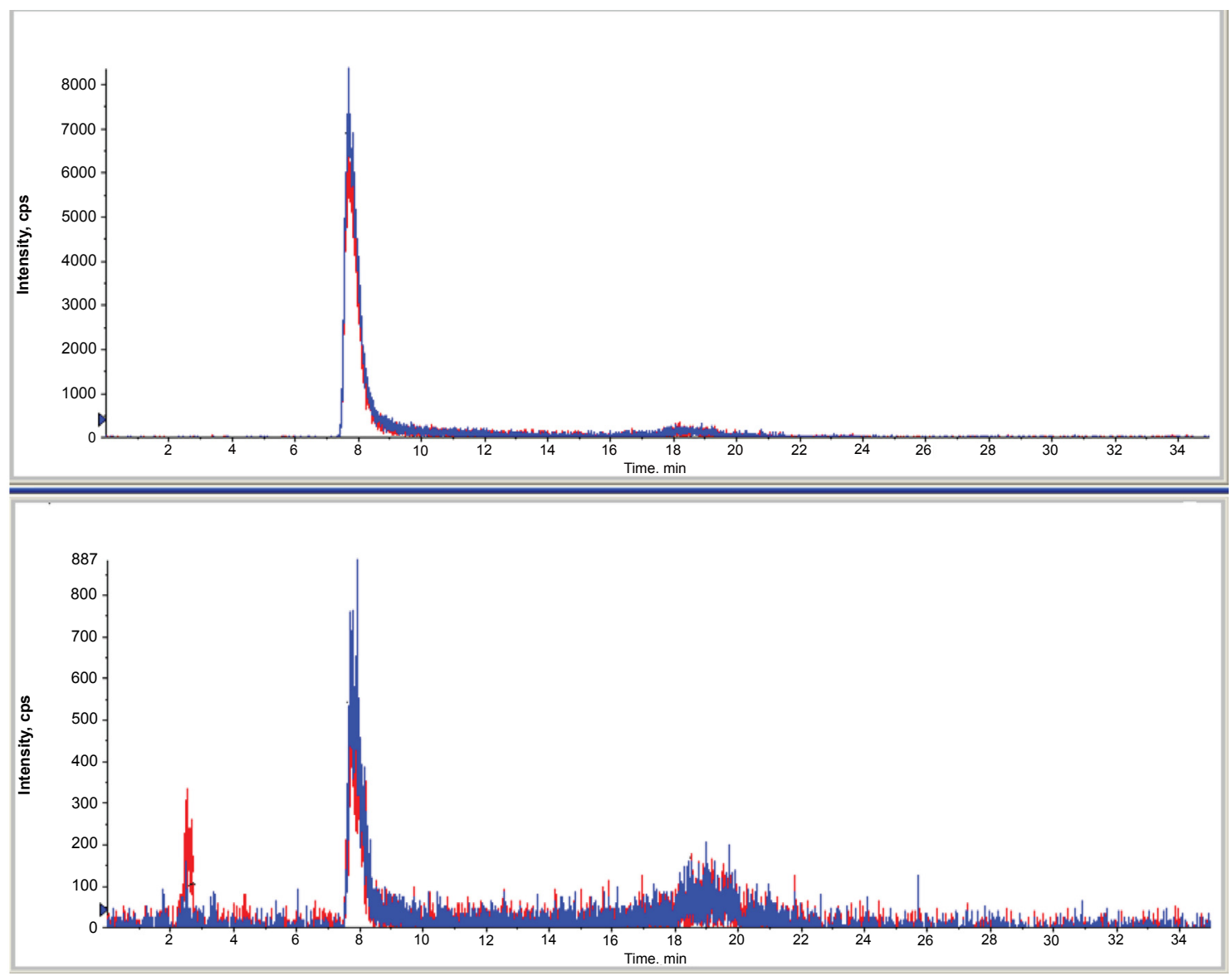

Figure 3: Chromatogram of a Standard solution of Staurosporine (top) and that of Salinispora M102 extract (bottom) monitored using two MRM transitions. 
Citation: Hewavitharana AK, Shaw PN, Ng YK, Fuerst JN (2009) Simple Screening Method for Staurosporine in Bacterial Cultures using Liquid Chromatography-Tandem Mass Spectrometry. J Bioanal Biomed 1: 001-004. doi:10.4172/1948-593X.1000001

peaks were absent from the blank extracts of sterile culture medium. Figure 3 shows the chromatogram of the standard and that of an extract (M102) monitored in MRM mode for two transitions $(467 \mathrm{~m} / \mathrm{z}$ ion $\rightarrow 338 \mathrm{~m} / \mathrm{z}$ ion and $467 \mathrm{~m} / \mathrm{z}$ ion $\rightarrow 130 \mathrm{~m} / \mathrm{z}$ ion). Both transitions were present (as two overlapping traces on both chromatograms) confirming the presence of Staurosporine in the extract. The confirmation in this case is based on matching four different parameters in the chromatograms of bacterial extracts to those in the chromatogram of the standard: the retention time, presence of the molecular ion and the presence of two fragments. This is a far superior confirmation compared to matching the retention time alone, common practise in chromatographic qualitative analysis. Chromatography of the standard was also monitored using MS2 mode (fragmentation monitored using the third quadrupole).

Four of the 15 Salinispora extracts screened contained Staurosporine. By an approximate estimation based on the calibration using the standard solution of Staurosporine the concentrations found in four strains analysed varied between 0.1 and 5 $\mu \mathrm{M}$. They can be ranked from the highest to lowest concentration of Staurosporine present as M102 > YKPC3 > SW15 > M413.

The on-column limit of detection for Staurosporine (estimated based on $3 \mathrm{x}$ baseline noise) was 5 pmol. Considering the extent of extract concentration and the volume injected $(20 \mu \mathrm{L})$ this equates to a Staurosporine concentration in the bacterial extract of $25 \mathrm{nM}$ or $11 \mathrm{ng} / \mathrm{mL}$. This level of sensitivity is inherent to MRM mode of MS detection which enables the efficient screening of bacteria such as Salinispora for the production of bioactive compunds. Extremely small concentrations of the compound are detected with a very high degree of specificity, with minimal sample preparation, within a very short time. The specificity of LC-MS-MS is crucial for the confirmation of the presence of Staurosporine in Salinispora. Compared to other methods available for screening of compounds in complex extracts, such as nuclear magnetic resonance (nmr) where isolation of the pure compound from the extract is required for confirmation, the method presented here is simple, more economical and rapid. The method of choice for screening so far has been the nmr (Park et al., 2006; Wu et al., 2006) which requires the isolation of the pure compound in substantial quantity. The method presented here is capable of confirming the presence of the compound with high degree of confidence.

The emphasis of this work was to develop a simple screening method for Staurosporine rather than to develop and validate a method for the quantitation of Staurosporine. However, the screening method presented here can be used as a semi-quantitative method and if required it can be further developed and validated to be a method of quantitation for Staurosporine. Although chromatographic quantitation with non-specific UV detection is available (Gurley et al., 1995) for Staurosporine, to date there are no reported methods using chromatography with mass spectrometric detection. The tandem mass spectrometric
MRM method developed in this study is capable of screening bacterial extracts for the presence of Staurosporine down to pmol levels, with confidence. Due to high specificity, it has the potential to screen any other plant extract for the presence of Staurosporine.

\section{References}

1. Bradshaw TD, Gescher A, Pettit GR (1992) Modulation by Staurosporine of phorbol-ester-induced effects on growth and protein-kinase-C localization in A549 human lung-carcinoma cells. Int J cancer 51: 144-148. »CrossRef » PubMed » Google Scholar

2. Funato N, Takayanagi H, Konda Y, Toda Y, Harigaya Y, et al. (1994) Absolute-configuration of Staurosporine by X-ray-analysis. Tetrahedron Lett 35: 1251-1254. »CrossRef » PubMed » Google Scholar

3. Gurley LR, Umbarger KO, Kim JM, Bradbury EM, Lehnert BE (1995) Development of a high-performance liquid chromatographic method for the analysis of Staurosporine. J Chromatogr B 670: 125-138. »CrossRef » PubMed » Google Scholar

4. Hachisu M, Hiranuma T, Koyama M, Sezaki M (1989) Antihypertensive compounds with potent protein-kinases inhibitory activity. Life Sci 44: 13511362. »CrossRef » PubMed » Google Scholar

5. Hewavitharana AK, Shaw PN, Kim TK, Fuerst JA (2007) Screening of rifamycin producing marine sponge bacteria by LC-MS-MS. J Chromatogr B 852: 362-366. »CrossRef » PubMed » Google Scholar

6. Jensen PR, Williams PG, Oh D-C, Zeigler L, Fenical W (2007) Speciesspecific secondary metabolite production in marine actinomtcetes of the genus Salinispora. Appl Environ Microbiol 73: 1146-1152. »CrossRef » PubMed » Google Scholar

7. Lin Y, Chrest FJ, Gabrielson EW (1992) Reversible G1 arrest of a human lung epithelial-cell line by Staurosporine. J Cell Physiol 152: 646-653. » CrossRef » PubMed » Google Scholar

8. Link JT, Raghavan S, Danishefski SJ (1995) Total synthesis of Staurosporine and Ent-Staurosporine. J Am Chem Soc 117: 552-553. »CrossRef » PubMed » Google Scholar

9. Ōmura S, Iwai Y, Hirano A, Nakagawa A, Awaya J, et al. (1977) A new alkaloid AM-2282 of Streptomyces origin. Fermentation, isolation and preliminary characterization. J Antibiot 30: 275-282. » CrossRef » PubMed » Google Scholar

10. Park HJ, Lee JY, Hwang IS, Yun BS, Kim BS, et al. (2006) Isolation and antifungal and antioomycete activities of Staurosporine from Streptomyces roseoflavus strain LS-A24. J Agric Food Chem 54: 3041-3046.» CrossRef » PubMed » Google Scholar

11. Schachtele C, Seifert R, Osswald H (1988) Stimulus-dependant inhibition of platelet-aggregation by the protein kinase-C inhibitors polymyxin- $\mathrm{B}, \mathrm{H}$ 7 and Staurosporine. Biochem. Biophys. Res Commun 151: 542-547. » CrossRef » PubMed » Google Scholar

12. Wu SJ, Fotso S, Li F, Qin S, Kelter G, et al. (2006) N-CarboxamidoStaurosporine and Selina-4(14),7(11)-diene-8,9-diol, new metabolites from a marine streptomyces sp. J Antibiot 59: 331-337. »CrossRef » PubMed » Google Scholar

13. Yamamoto S, Kiyoto I, Aizu E, Nakadate T, Hosoda Y, et al. (1989) Differential inhibition by Staurosporine, A potent protein kinase-C inhibitor, of 12-o-tetradecanoylphorbol-13-acetate-caused skin tumor promotion, epidermal ornithine decarboxylase induction, hyperplasia and inflammation. Carcinogenesis 10: 1315-1322. » CrossRef » PubMed » Google Scholar

14. Yang SW, Cordell GA (1997) Deuterium labeling and mass fragmentation studies of Staurosporine. J Nat Prod 60: 236-241. » CrossRef » PubMed » Google Scholar 